\title{
A linear recursive state of power estimation for fusion model component analysis with constant sampling time.
}

\author{
LI, B., WANG, S., FERNANDEZ, C., YU, C., SHI, W. and FAN, Y.
}




\title{
A Linear Recursive State of Power Estimation for Fusion Model Component Analysis with Constant Sampling Time
}

\author{
Bowen Li $^{l}$, Shunli Wang ${ }^{1, *}$, Carlos Fernandez ${ }^{2}$, Chunmei Yu ${ }^{l}$, Weihao Shi ${ }^{l}$, Yongcun Fan ${ }^{l}$ \\ ${ }^{1}$ School of Information Engineering, Southwest University of Science and Technology, Mianyang \\ 621010, China; \\ ${ }^{2}$ School of Pharmacy and Life Sciences, Robert Gordon University, Aberdeen, AB10-7GJ, UK \\ "E-mail: wangshunli@swust.edu.com
}

Received: 1 May 2021 / Accepted: 26 June 2021 / Published: 10 August 2021

\begin{abstract}
The state of power of lithium-ion batteries, as the main product of choice for electric and hybrid electric vehicle energy storage systems, is one of the precise feedback control parameters for the battery management system. The proposed research establishes a method for the analysis of charging and discharging constitutive factors under the sampling time, realizes the online identification of parameters by building an adaptive forgetting factor recursive least-squares method based on the Thevenin model, and uses the online parameters to achieve an effective characterization of the power state under voltage and current limitations. The results demonstrate that the accuracy error of online parameter identification is less than $0.03 \mathrm{~V}$. Combining the analysis of charging and discharging constitutive factors undersampling time with the fusion model of voltage and current limitation makes the power state estimation more reliable and accurate. The results demonstrate that the power state estimation error in the discharging state is less than $8 \%$.
\end{abstract}

Keywords: state of power; lithium-ion batteries; energy storage systems; charging and discharging constitutive factors; online identification; fusion model of voltage and current limitation

\section{INTRODUCTION}

With the continued development of the new energy vehicle industry, traditional batteries are difficult to adapt to the industry's development[1-3]. Lithium-ion batteries have become a popular choice for Electric Vehicles (EVs) and Hybrid Electric Vehicles (HEVs) due to their high energy density, high output power, and low pollution [4, 5]. However, lithium-ion batteries, due to their own defects, improper use can easily lead to safety hazards [6,7]. Therefore, the state index of lithium-ion is a mandatory reference quantity and an important limiting quantity, and the ability to accurately predict the state index will directly affect the reliable use of the vehicle $[8,9]$. 
The state of power (SOP) of a lithium-ion battery, an important indicator characterizing the energy output of a vehicle, is defined as the maximum output power that the battery can deliver for a given duration [10,11]. This indicator is usually strongly coupled with the voltage, current, and resistance of the battery $[12,13]$. Therefore, the effective estimation of SOP is inseparable from the effective tracking of the above parameters. It is not difficult to find that the maximum power output of a new energy vehicle is reflected by the SOP, which provides owners with reliable power information to optimize the energy output of the vehicle.

Combining the previous work, it is necessary to establish a battery model that can be quickly constructed to analyze the resistance and capacitance characteristics of the battery[14-16]. Currently, there are three main types of battery models used to describe the new energy characteristics of batteries: electrochemical models (EM), equivalent circuit models (ECM), and electrochemical impedance models (EIM) [17-19]. Yang, X.considered the solid-phase lithium-ion diffusion equation based on the electrode averaging model of the battery and used a finite difference method for a simplified representation of EM[20]. Zhang, X. analyzed the characteristics of batteries by introducing electrochemical performance to characterize the ECM, and build an improved pseudo-two-dimensional model to describe it[21]. In addition, Chen, N. used electrochemical impedance spectroscopy to analyze their time-domain circuit model in the effect of constant phase elements to construct an equivalent circuit model based on electrochemical impedance[22]. The above work not only relies on a large amount of data but also has a high computational complexity [23, 24]. Obviously, online parameter identification is difficult to analyze its higher-order state characterization due to its complexity and accuracy[25, 26]. The ECM model has the characteristics of simplicity, ease of calculation, and ability to reliably characterize its resistance and capacitance characteristics, which can satisfy the state parameter analysis of the battery.

Based on the previous work, SOP calculations are often determined by the coupling relationship between voltage, circuit, and other resistive and capacitive characteristics parameters[27]. A joint estimation scheme for the state of charge (SOC) and SOP lithium-ion batteries for electric vehicles based on the fractional-order model is proposed [28]. Cheng, Z. proposed an intelligent algorithm based on SOC, battery voltage, and voltage combination constraint[29]. Yang, L. established a battery model with multi-parameter dynamic open-circuit voltage and a battery long-term power demand (LTPD) prediction model to achieve long-term prediction[30]. Dual polarization (DP) ternary lithium-ion battery model to estimate SOC and SOP joint at a plurality of constraints, and the Extended Kalman Filter (EKF) algorithm to improve the accuracy of state. However, none of the above algorithms consider the power output representation at long sampling periods, which is not conducive to the effective characterization of the vehicle at long power output representation.

It is worth mentioning that in the study of literature [31], a linear recursive under fusion model is proposed to analyze the SOP output. The main focus of this method is to realize the analysis of the error effects of parameters on SOP under different algorithms by establishing a full-parameter estimation system, and on this basis, to realize the state evaluation under constant power preset observation. From the conclusion, it is easy to conclude that the coupling relationship of the model parameters has a large impact on the battery SOP estimation, but there is a large error in the peak power estimation for a long time. Therefore, analyzing the model state under unknown sampling time and using it as a basis to determine the power output of the battery at different durations becomes the main idea of this study. 
Combined with the above work, the implementation of this algorithm should be both complex and feasible. In this study, a method for analysis of charging and discharging constitutive factors undersampling time is established, online identification of parameters is realized by establishing an adaptive forgetting factor recursive least-squares (AFFRLS) method based on Thevenin model, and effective characterization of SOP under voltage and current limitations is achieved using online parameters. Firstly, the adaptive online parameter identification is constructed by analyzing the battery model. Secondly, the power occupation ratio is judged by analyzing the charging and discharging components of the battery under the sampling time and setting the coupling reference quantity. Finally, a fusion model based on voltage and current limits is constructed for linear recursive estimation.

\section{MATHEMATICAL ANALYSIS}

\subsection{Lithium-ion battery modeling}

An important way to describe the parameters of a lithium-ion battery is achieved through model building. Considering the computational time consumption and memory footprint of the algorithm in relation to the complexity of the model, the Thevenin model was chosen to describe the operating characteristics. The lithium-ion battery model is shown in Fig. 1.

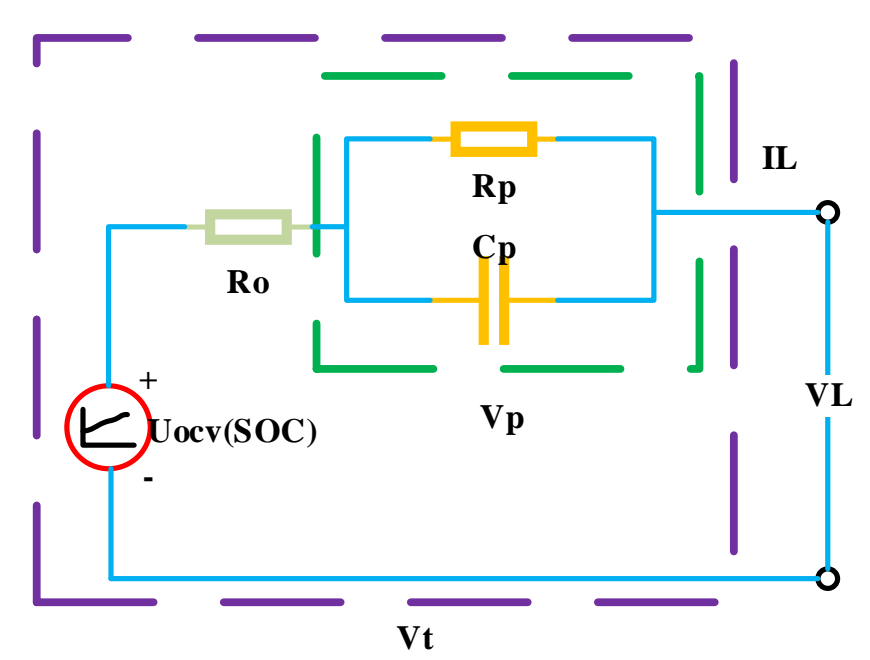

Figure 1. The Thevenin model

Among them, the ECM shown in the figure is divided into four main parts: (i) the battery terminal voltage Uoc (SOC) is the fitted curve obtained by the HPPC experiment; (ii) ohmic resistance R0 represents the ohmic effect of the battery; (iii) polarization resistance $\mathrm{Rp}$ and polarization capacitance Cp represent the polarization effect of the resistor; (iv) OCV VL represents the load voltage under battery load conditions. In addition, $\mathrm{Vp}$ represents the voltage under the polarization effect; Vt represents the ohmic effect and polarization effect voltage; IL is the discharge current of the lithium battery.

Thus, the model of this circuit can be represented as 


$$
\left\{\begin{array}{l}
\chi_{k}=A_{k-1} \chi_{k-1}+B_{k-1} I_{L, k-1} \\
V \mathrm{t}=f\left(\chi_{k}, I_{L, k}\right)
\end{array}\right.
$$

Where

$$
\left\{\begin{array}{l}
\chi_{k}=\left[\begin{array}{ll}
V_{p, k} & S O C_{k}
\end{array}\right]^{T} \\
A_{k-1}=\left[\begin{array}{ll}
\varepsilon_{k-1} & 0 \\
0 & 1
\end{array}\right] \\
B_{k-1}=\left[\begin{array}{ll}
R_{p, k-1}\left(1-\varepsilon_{k-1}\right) & \frac{T s}{3600 Q_{c}}
\end{array}\right]^{T} \\
\varepsilon_{k-1}=e^{\frac{-T s}{R_{p, k-1} C_{p, k-1}}} \\
f\left(\chi_{k}, I_{L, k}\right)=U_{o c v(S O C), k}+R_{0, k} I_{L, k}+V_{p, k}
\end{array}\right.
$$

$T s$ is the unit sampling time and $Q c$ is the rated capacity of the battery. Generally, the characterization of the lithium-ion battery model is based on the OCV-SOC curve. Obviously, lithiumion batteries exhibit different OCVs at different temperatures. The relationship of SOC-OCV for lithiumion batteries at different temperatures is shown in Fig. 2.

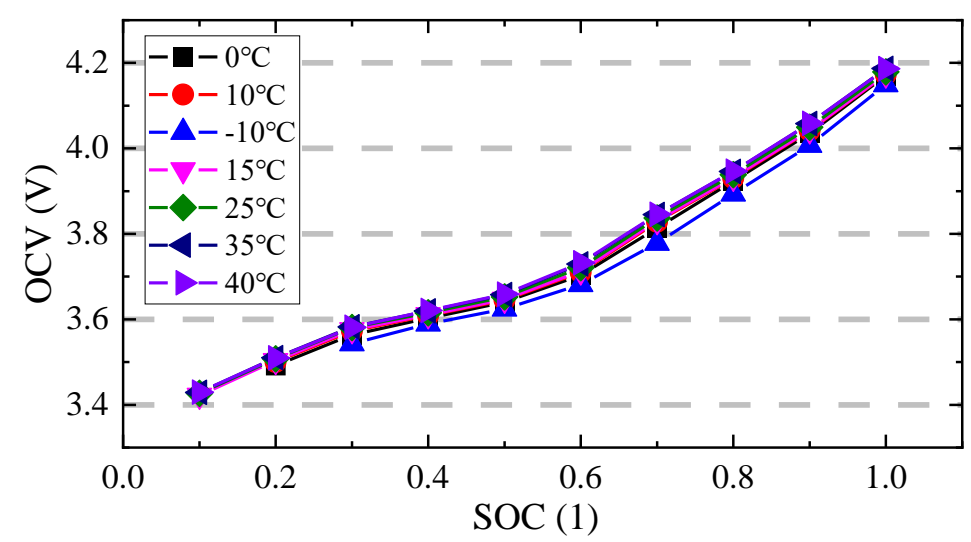

Figure 2. The relationship of SOC-OCV for lithium-ion batteries at different temperatures

Obviously, lithium-ion batteries exhibit different OCVs at different temperatures. It can be seen from the figure that within a certain temperature range, the OCV value also increases as the ambient temperature increases. The accurate OCV-SOC curve is an indispensable part of the accurate parameter identification process.

\subsection{Online parameter identification based on AFFRLS}

The parameter characteristics of lithium-ion batteries acquired in practice are fluctuating and changing. The least-squares (LS) method allows correction and analysis of fluctuating data in the time domain for online parameter identification. Due to its accuracy, Adaptive Recursive Forgotten Factor Least Squares (AFFRLS) is introduced to continuously correct the factor according to the error variance 
to obtain the best effect change curve. The flow chart of the AFFRLS algorithm for lithium-ion batteries is shown in Fig. 3.

Among them, $X(t)$ represents the working condition of the battery; $Y(t)$ represents the parameter under online recognition; $e(t)$ represents the error; the part indicated by the dotted line represents the initial value introduction. Import the experimental working condition data, and obtain the online parameter identification value directly through the AFFRLS algorithm.
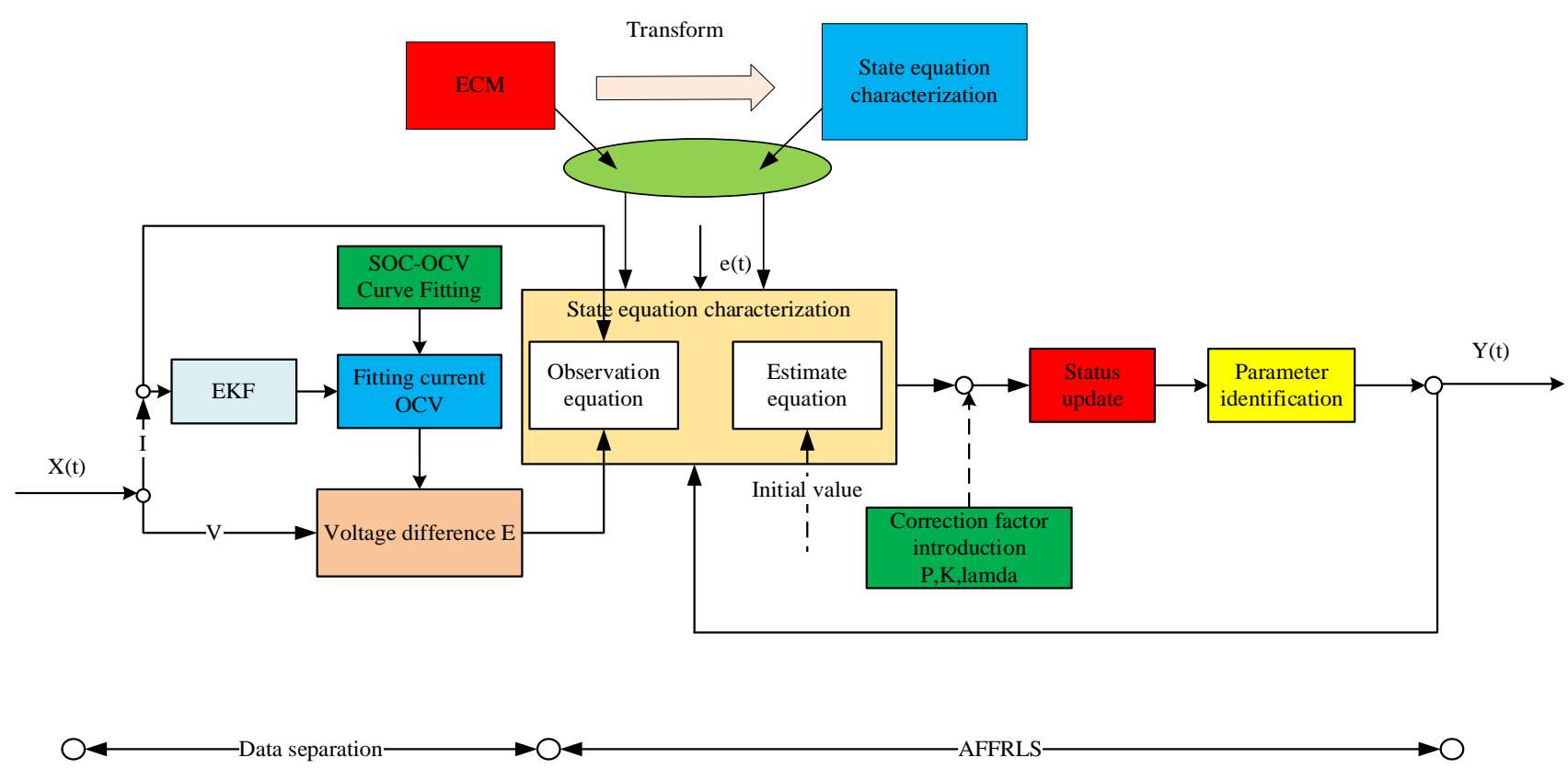

Figure 3. The flow chart of the AFFRLS algorithm for lithium-ion batteries

Of course, accurate analysis of the operating characteristics of lithium-ion batteries is inseparable from the model's equation of state analysis. In combination with equations (1) and (2), Eq. 3 is discretized to reflect the characteristics of the battery.

$$
E_{k} s=U_{L, k} s-U_{o c v(S O C), k} s=-I_{L, k} s\left(R_{0, k}+\frac{R_{p, k}}{1+R_{p, k} C_{p, k} s}\right)
$$

Thus, the identification of ECM parameters of lithium-ion batteries is converted into expressions of transfer functions related to voltage and current. Eq. 3 can be rewritten as Eq. 4.

$$
G_{k} s=\frac{E_{k} s}{I_{L, k} s}=-\frac{\frac{R_{p, k}+R_{0, k}}{R_{p, k} C_{p, k}}+R_{0, k} s}{s+\frac{1}{R_{p, k} C_{p, k}}}
$$

Bilinear changes $s=\frac{2}{T} \times \frac{1-z^{-1}}{1+z^{-1}}$ introduce time-domain signals into frequency domain signals for analysis, then Eq. 4 can be characterized by Eq. 5 . 


$$
\mathrm{G}\left(z^{-1}\right)=\frac{E(k)}{I_{L}(k)}=\frac{\theta_{2, k}+\theta_{3, k} z^{-1}}{1-\theta_{1, k} z^{-1}}
$$

Among them,

$$
\left\{\begin{array}{l}
\theta_{1, k}=\frac{2 R_{p, k} C_{p, k}-T}{2 R_{p, k} C_{p, k}+T} \\
\theta_{2, k}=\frac{2 R_{0, k} R_{p, k} C_{p, k}+T\left(R_{p, k}+R_{0, k}\right)}{2 R_{p, k} C_{p, k}+T} \\
\theta_{3, k}=\frac{2 R_{0, k} R_{p, k} C_{p, k}-T\left(R_{p, k}+R_{0, k}\right)}{2 R_{p, k} C_{p, k}+T}
\end{array}\right.
$$

By tracking and analyzing the internal voltage and working current of the battery in real-time, it is easy to analyze the changes of battery characteristic parameters every moment. Rewrite Eq. 6 into Eq. 7 to obtain the change of parameters at each moment.

$$
\left\{\begin{array}{l}
R_{0, \mathrm{k}}=\frac{\theta_{2, k}-\theta_{3, k}}{\theta_{1, k}+1} \\
R_{\mathrm{p}, \mathrm{k}}=\frac{\theta_{2, k}-\theta_{3, k}}{\theta_{1, k}+1}-R_{0, \mathrm{k}} \\
C_{\mathrm{p}, \mathrm{k}}=\frac{1}{2 R_{\mathrm{p}, \mathrm{k}}} \frac{T\left(1+\theta_{1, k}\right)}{1-\theta_{1, k}}
\end{array}\right.
$$

The LS method uses the square norm of the discrete function as a metric to obtain the identification parameters. For the representation of continuous and repeated working condition parameters, it can be expressed by Eq. 8 .

$$
\left\{\begin{array}{l}
E_{2}=\theta_{1,1} E_{1}+\theta_{2,1} I_{L, 2}+\theta_{3,1} I_{L, 1}+e_{1}(k) \\
E_{3}=\theta_{1,2} E_{2}+\theta_{2,2} I_{L, 3}+\theta_{3,2} I_{L, 2}+e_{2}(k) \\
\vdots \quad \vdots \quad \vdots \quad \vdots \quad \vdots \quad \\
E_{k}=\theta_{1, k} E_{k-1}+\theta_{2, k} I_{L, k}+\theta_{3, k} I_{L, k-1}+e_{3}(k)
\end{array}\right.
$$

The data of experimental working conditions often have the influence of noise, which makes the data fluctuate. In consideration of the influence of noise, a matrix $e$ is introduced to characterize this interference. To simplify the expression, rewrite Eq. 8 into Eq. 9.

$$
E_{k}=\varphi_{k} \sigma_{k}^{T}+e_{k}
$$

Wherein, $\varphi(k)$ is the observation vector, $\sigma(k)$ is the parameter vector to be estimated. According to the relationship between the system input and the system output, the n-dimensional representation of the matrix can be obtained after changing and processing the coefficients of the matrix, as described above.

$$
\left\{\begin{array}{l}
J\left(\sigma_{k}\right)=\left(E_{k}-\varphi_{k} \sigma_{k}\right)^{T}\left(E_{k}-\varphi_{k} \sigma_{k}\right) \\
\frac{\partial J}{\partial \sigma_{k}}=\frac{\partial}{\partial \sigma_{k}}\left[\left(E_{k}-\varphi_{k} \sigma_{k}\right)^{T}\left(E_{k}-\varphi_{k} \sigma_{k}\right)\right]=0
\end{array}\right.
$$


The LS method is to optimize data under fluctuating conditions. Therefore, the parameter correction at each moment should be based on the reliable last-minute correction value and the changing conditions. Combining Eq. 9 and Eq. 10, the modified parameter correction relationship can be obtained, as shown in Eq. 11.

$$
\sigma_{k}=\left(\left(\varphi_{\mathrm{k}}^{T} \varphi_{\mathrm{k}}\right)^{-1} \varphi_{\mathrm{k}}^{T} E_{k}\right)
$$

With the increase of iteration times of algorithm data, the classical LS method makes the gain $K$ and matrix variance $P$ becomes smaller and smaller, which weakens the optimization effect of subsequent calculation. Recursive Least Square Algorithm with Forgetting Factor (FFRLS) increases the forgetting factor $\lambda$ to weaken the influence of the previous iteration and improve the online estimation ability of the algorithm. Among them, the forgetting factor is often set at 0.96 to 1 according to a large number of experiments. The prediction update estimation process of the FFRLS algorithm is shown in Eq. 12.

$$
\left\{\begin{array}{l}
\sigma_{k+1}=\sigma_{k}+K_{k+1}\left[E_{k+1}-\varphi^{T}{ }_{k+1} \sigma_{k}\right] \\
K_{k+1}=P_{k} \varphi_{k+1}\left[\varphi_{k+1}^{T} P_{k} \varphi_{k+1}+\lambda_{k}\right]^{-1} \\
P_{k+1}=\lambda_{k}^{-1}\left[I-K_{k+1} \varphi_{k+1}^{T}\right] P_{k}
\end{array}\right.
$$

Correspondingly, for the analysis of a large number of continuously changing data, the fixed and unchanging impression factor cannot be changed accordingly, which also brings parameter identification errors to a certain extent. When the correction ability of the forgetting factor reaches the limit, the system identification will also accumulate errors.

The AFFRLS algorithm obtains an algorithm tracking effect more in line with the actual changes of the working conditions through the continuous modification of the forgetting factor. An equation to calculate the variable forgetting factor to achieve the aforementioned goal is expressed by Eq. 13 .

$$
\left\{\begin{array}{l}
\lambda_{k}=\operatorname{limit}\left(\lambda_{\min }+\left(1-\lambda_{\min }\right) g^{w_{k}}\right) \\
w_{k}=\operatorname{round}\left(\frac{e_{k}}{e_{\text {base }}}\right)^{2}
\end{array}\right.
$$

Among them, $\lambda_{\min }$ is the minimum value of the forgetting factor. A large amount of experimental data combined with ECM proves that the AFFRLS algorithm is accurately and quickly corrected when the forgetting factor is set in the range of 0.98 to $1, \lambda_{\min }$ is 0.98 . limit indicates that the reference object is restricted so that its maximum value does not exceed $1 .{ }^{g}$ is the sensitivity coefficient. ${ }^{g}$ can be chosen to be any value between 0 and 1 , indicating the sensitivity of the forgetting factor to errors. When $g$ approaches 1 , the forgetting factor slowly changes from 1 to 0.98 , resulting in a slow response parameter recognition speed. Conversely established. ${ }^{e_{k}}$ is the error at time $\mathrm{k}$, and $e_{\text {base }}$ is the allowed error reference; ${ }_{b a s e}$ is usually based on expected errors. When the identification parameter error is less than $e_{b a s e}$, the identification parameter is considered stable, and $\lambda$ changes to a larger value. Conversely established. The function $\operatorname{round}(n)$ represents the integer closest to $n$. Thereby, changing genetic factors can be captured. 


\subsection{State of Power estimation under fusion algorithm}

The estimation of SOP is inseparable from the analysis of voltages and currents. There are two common algorithmic reference schemes. (i) SOP estimation based on voltage limitation; (ii) SOP estimation based on current limitation. It is difficult to estimate the battery effectively with a single cell limit. Considering the operating characteristics of the battery, a fusion estimation model under voltage and current limits is proposed to estimate SOP.

This study innovatively proposes a charge/discharge component analysis method under consideration of sampling time, and the implementation principle is shown in Fig. 4. Separate prediction of time is achieved by analyzing the causes of voltage and current limitations (the specific calculation is shown in Eq. 21).
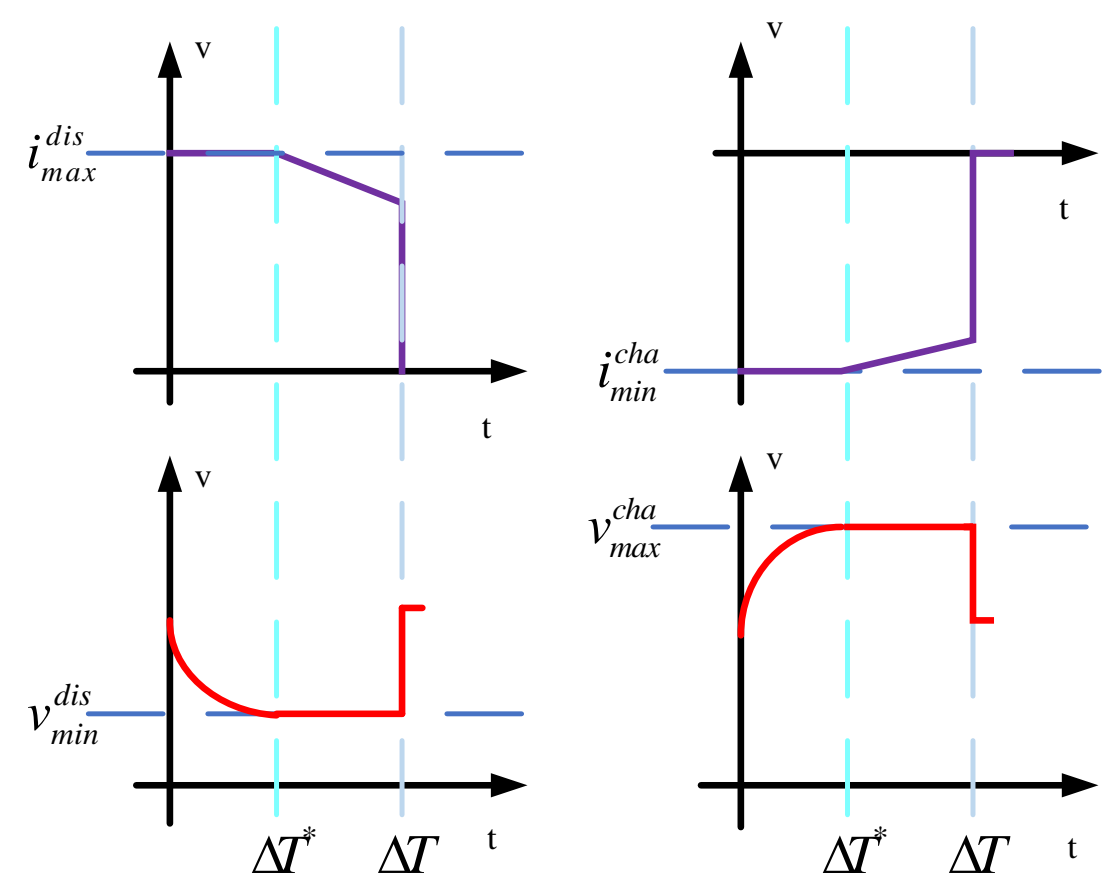

Figure 4. Analysis of charge and discharge components under-sampling time

Among them, $\Delta T^{*}$ is the time under current limitation; $\Delta T$ is the sampling time. From the aforementioned work, the fusion algorithm is based on a mixed analysis of two situations. It is indispensable to introduce the SOP algorithm based on voltage limitation and current limitation.

SOP estimation based on voltage limit conditions is assumed that the terminal voltage of the battery $v$ is in the range from $v_{\min }$ to $v_{\max }$. Then Eq. 1 can be rewritten as a state-space representation, as shown in Eq. 14. 


$$
\left\{\begin{array}{l}
\chi_{k+K_{T}}=A_{k}^{K_{T}}+\left(\sum_{n=1}^{K_{T}} A_{k}^{n-1} B_{k}\right) I_{L, k} \\
K_{T}=\frac{\square T}{T s}
\end{array}\right.
$$

Where $\square T$ represents the sampling time width; $\mathrm{K}_{\mathrm{T}}$ is the change in unit time at the sampling time. Therefore, for each time sampling $\mathrm{k}$, assuming that the terminal voltage is equal to the predefined limit, the maximum/minimum discharge/charge current of the upcoming time is calculated by Eq. 15 .

$$
\left\{\begin{array}{c}
I_{d i s, k+K_{T}}=-\frac{v_{\min }-U_{o c v(S O C), k+K_{T}}+\varepsilon_{k+K_{T}} K_{T} V_{p, k+K_{T}}}{\frac{\eta K_{T} T s}{Q_{c, k}} \times\left.\frac{\partial U_{o c v(S O C)}}{S o C}\right|_{S O C=S O C_{k+K_{T}}}+R_{0, k+K_{T}}+R_{p, k+K_{T}}\left(1-\varepsilon_{k+K_{T}}\right) \sum_{n=1}^{K_{T}} \varepsilon_{k+K_{T}}{ }^{n-1}} \\
I_{c h a, k+K_{T}}=-\frac{v_{\max }-U_{o c v(S O C), k+K_{T}}+\varepsilon_{k+K_{T}}{ }^{K_{T}} V_{p, k+K_{T}}}{\frac{\eta K_{T} T s}{Q_{c, k}} \times\left.\frac{\partial U_{o c v(S O C)}}{S o C}\right|_{S O C=S O C_{k+K_{T}}}+R_{0, k+K_{T}}+R_{p, k+K_{T}}\left(1-\varepsilon_{k+K_{T}}\right) \sum_{n=1}^{K_{T}} \varepsilon_{k+K_{T}}{ }^{n-1}}
\end{array}\right.
$$

Among them, $I_{d i s}$ is the characterization of the battery discharge current under the voltage limit; $I_{c h a}$ is the battery characterization of the charge current under the voltage limit. Obviously, the SOP prediction of the lithium-ion battery at this time can be expressed by Eq. 16 .

$$
\left\{\begin{array}{l}
S O P_{d i s, k+K_{T}}^{v}=v_{\min } I_{k+K_{T}}^{d i s} \\
S O P_{c h a, k+K_{T}}^{v}=v_{\max } I_{k+K_{T}}^{c h a}
\end{array}\right.
$$

Among them, $S O P_{d i s}^{v}$ represents the battery discharge SOP under the voltage limit discharge; $S O P_{c h a}^{v}$ represents the battery discharge SOP under the voltage limit charge. In summary, it is possible to achieve a reliable prediction of SO under-voltage limitations.

SOP estimation based on current limit conditions is assumed that the terminal voltage of the battery $i$ is in the range from $i_{\min }$ to $i_{\max }$. Based on the previous work, the voltage is used as the unknown reference value, and the calculation and analysis are carried out in combination with Eq. 1.

$$
E_{k+K_{T}}=-\varepsilon_{k+K_{T}} K_{T} V_{p, k+K_{T}}+\left\{\sum_{j=1}^{j=K_{T}}\left[-\varepsilon_{k+K_{T}}{ }^{K_{T}-j} R_{p, k+K_{T}}\left(1-\varepsilon_{k+K_{T}}\right)\right]-R_{0, k+K_{T}}\right\} i_{\lim }
$$

Among them, $i_{\text {lim }}$ is related to the load current of the battery model. Obviously, the SOP prediction of the lithium-ion battery at this time can be expressed by Eq. 18 .

$$
\left\{\begin{array}{l}
S O P_{d i s, k+K_{T}}^{i}=i_{\min } V_{k+K_{T}}^{d i s} \\
S O P_{c h a, k+K_{T}}^{i}=i_{\max } V_{k+K_{T}}^{c h a}
\end{array}\right.
$$

In fact, these two cases reflect the battery's operating characteristics more unilaterally. Therefore, consider combining the two conditions and use fusion to analyze the battery characteristics. For each sampling segment selected for analysis, the time when the battery charge/discharge status changes from constant current to constant voltage should be determined first. For this reason, it is assumed that the current is equal to its maximum/minimum discharge/charge. In this case, the terminal voltage is equal to the predetermined $v_{\min }$ (or $v_{\max }$ ) limit discharge (or charge). Since the input current is assumed to be constant, the terminal voltage can be written as Eq. 19. 


$$
\left\{\begin{array}{l}
v_{\text {min }}=U_{o c v(S O C), k+K_{T 1}}-V_{p, k+K_{T 1}} \varepsilon_{k+K_{T 1}} K_{T 1}-\left[R_{0, k+K_{T 1}}+R_{p, k+K_{T 1}}\left(1-\varepsilon_{k+K_{T 1}}^{K_{T 1}}\right)\right] i_{\max }^{d i s} \\
v_{\text {max }}=U_{o c v(S O C), k+K_{T 1}}-V_{p, k+K_{T 1}} \varepsilon_{k+K_{T 1}} K_{T 1}-\left[R_{0, k+K_{T 1}}+R_{p, k+K_{T 1}}\left(1-\varepsilon_{k+K_{T 1}}^{K_{T 1}}\right)\right] i_{\min }^{c h a}
\end{array}\right.
$$

Where $K_{T^{*}}$ represents the time sample of constant current charge/discharge. Therefore, Eq. 20 can be used to calculate $T^{*}$.

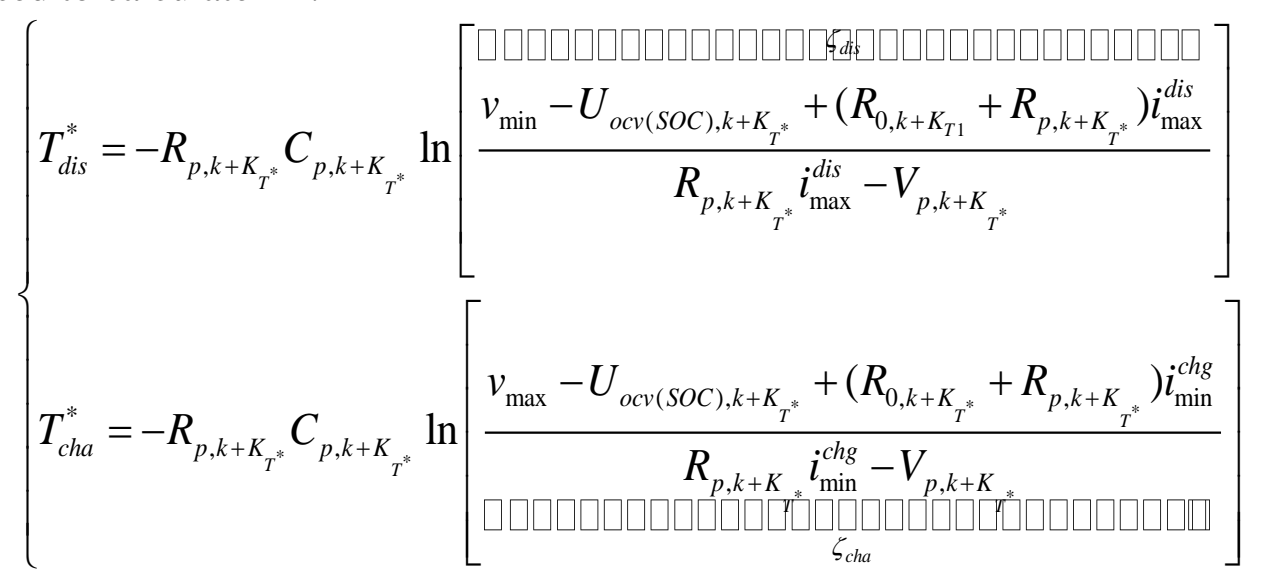

Therefore, the analysis of different constant current and constant voltage batteries can be obtained as shown below.

$$
\left\{\begin{array}{l}
\text { Constant Current: } \zeta \leq \varepsilon_{k+K_{T 1}}^{K_{T 1}} \Rightarrow \mathrm{T}^{*}=\mathrm{T} \\
\text { Constant Voltage: } \zeta>1 \Rightarrow \mathrm{T}^{*}=0 \\
\text { Fusion Model : } \varepsilon_{k+K_{T 1}}^{K_{T 1}}<\zeta \leq 1 \Rightarrow \mathrm{T}^{*}=-R_{p, k+K_{T 1}} C_{p, k+K_{T 1}} \ln \zeta
\end{array}\right.
$$

Based on the in-time samples, calculate the upcoming sequence of available power values, and use the equation to time-sample within the prediction range. Based on the analysis of the foregoing work, the minimum amplitude of the charging and discharging conditions of each sampling segment is expressed by Eq. 22.

$$
\left\{\begin{array}{l}
S O P_{d i s, k}=\min \left(S O P_{d i s, k+1}, S O P_{d i s, k+2}, \cdots, S O P_{d i s, k+K_{T}}\right) \\
S O P_{c h a, k}=\max \left(S O P_{c h a, k+1}, S O P_{c h a, k+2}, \cdots, S O P_{c h a, k+K_{T}}\right)
\end{array}\right.
$$

Considering the battery situation at each sampling stage under long-term fluctuating operating conditions, by analyzing the battery SOP at every other sampling point, it is obvious that the battery's charging state or discharging state in the current stage can be obtained.

\section{EXPERIMENTAL ANALYSIS}

\subsection{Test platform construction and data acquisition}

The accurate prognosis of lithium-ion batteries is achieved through a reliable experimental testing and analysis process. Secondly, the ambient temperature has a strong influence on the state of the battery. Therefore, this study is mainly based on the analysis under constant temperature conditions. The instruments used in this test are a high-power charge/discharge tester for power batteries (ct-40165V100a-tfa), a three-layer independent temperature-controlled test chamber, and other supporting 
experimental equipment (bt-331c). The experimental data testing process of the research object (lithiumion battery) is shown in Fig. 5.

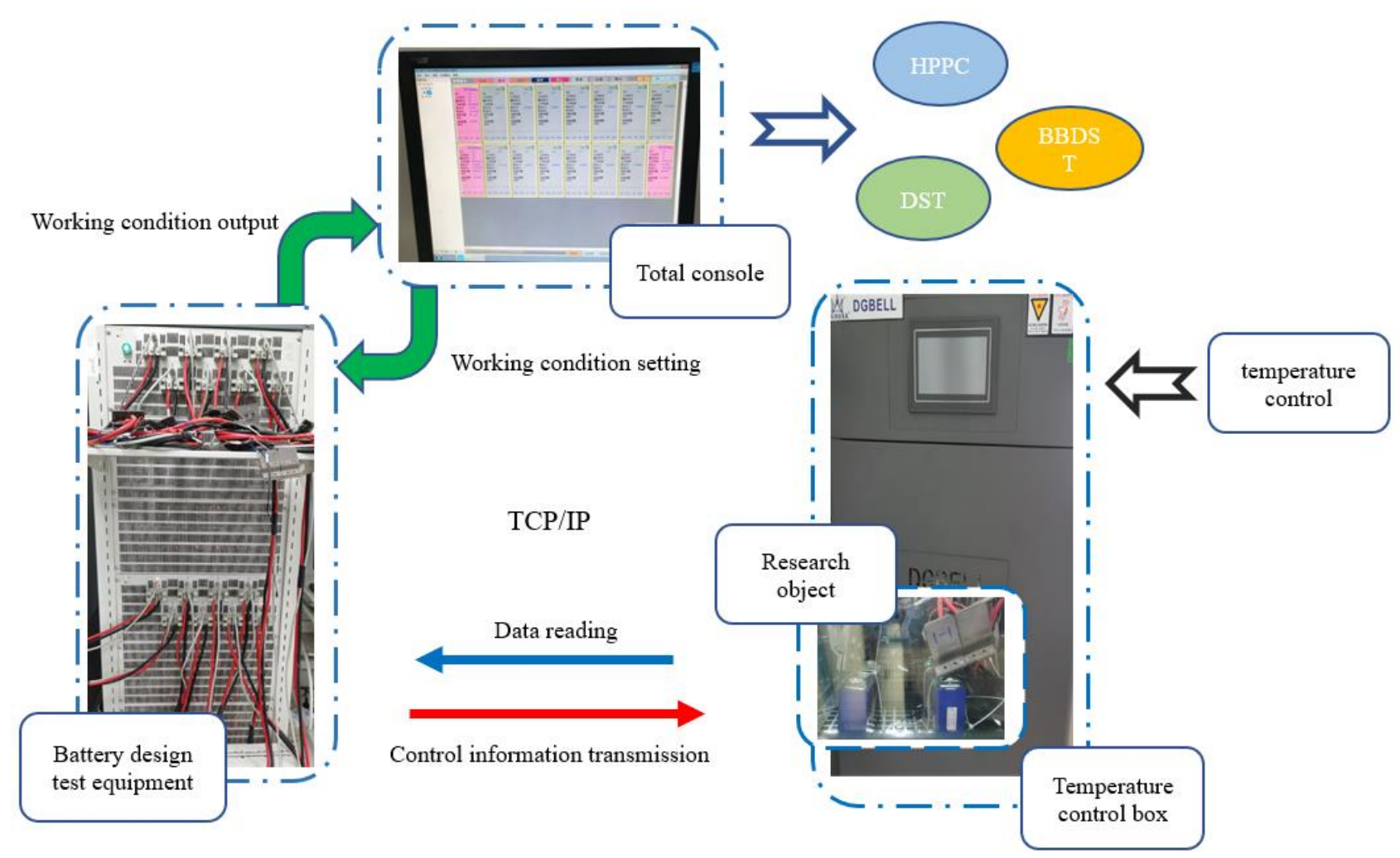

Figure 5. The test procedure for observation objects

As can be seen from the figure, the above study was conducted on a ternary lithium-ion battery (capacity 72.2Ah). The purpose of this study is to realize the reliable application of the battery in the environment, and the dynamic test analysis application is indispensable. Beijing Bus Dynamic Stress Test (BBDST) working conditions and Dynamic Stress Test (DST) working conditions are selected to verify the accuracy of online identification and the tracking effect of the fusion model algorithm. BBDST describes the power of the new energy bus under different driving operations The BBDST describes the power output characteristics of the new energy bus under different driving operations, and the DST describes the continuous peak discharge characteristics of the new energy bus at a constant time. Among them, the BBDST operating characteristic diagram and the DST operating characteristic diagram are represented in Fig. 6. 


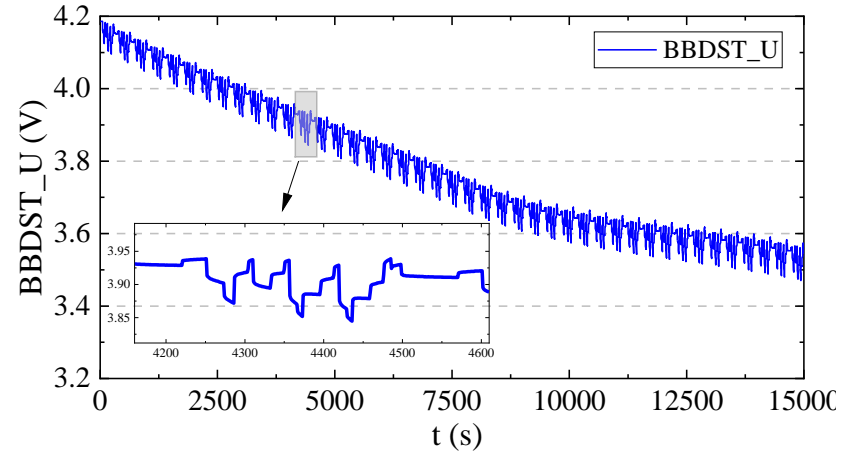

(a) BBDST working condition voltage global curve

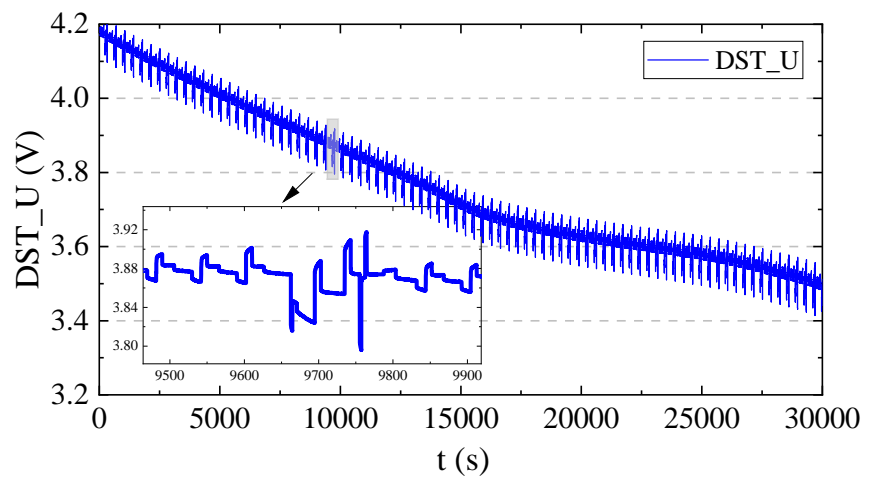

(c) DST working condition voltage global curve

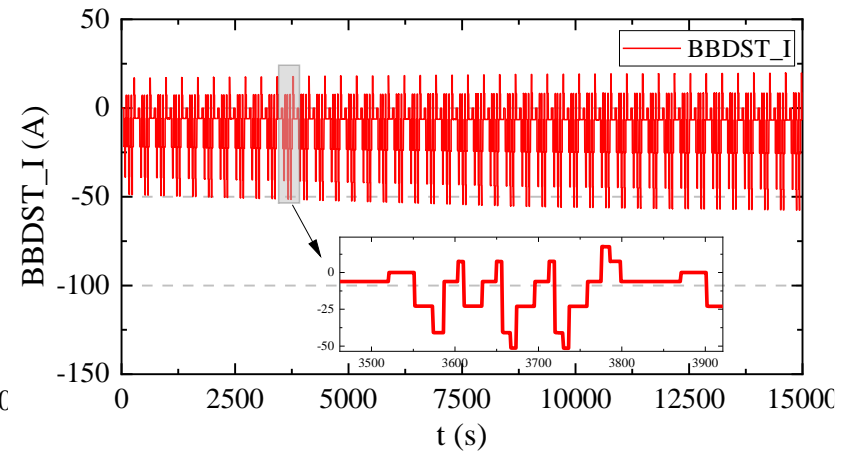

(b) BBDST working condition current global curve

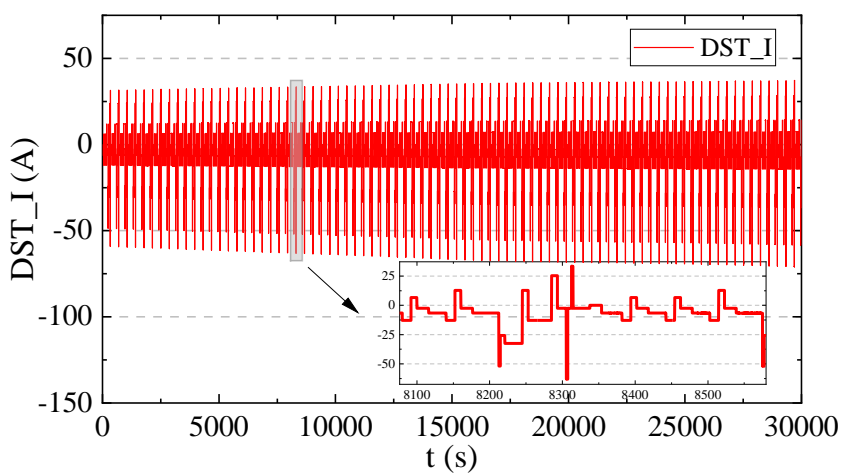

(d) DST working condition current global curve

Figure 6. Voltage and current curves of dynamic stress test conditions

As can be seen from the graph, the voltage and current variation under DST condition is huge compared to BBDST. It is worth mentioning that when the lithium-ion battery is tested under constant power condition, the battery cannot maintain a stable power output as the voltage decreases under the duration discharge point, which will lead to large fluctuations in the working current of the battery under different durations. Therefore, the study mainly analyzes the battery state of the former $70 \%$ SOC for research and analysis of its battery operating state.

\subsection{Working condition demonstration effect}

The operating characteristics of electric and hybrid vehicles vary considerably under driving conditions. Performance analysis of long-term lithium-ion batteries usually relies on online identification to characterize their operating characteristics. It is particularly important to obtain accurate dynamic parameter variation curves for lithium-ion batteries.

As can be seen in Fig. 3, the analysis using the RLS algorithm is predicated on obtaining the difference between the true voltage and the fitted voltage of the battery operating state. Therefore, in this paper, the SOC-OCV curve of the battery is obtained by analyzing the pulse discharge characteristics under constant temperature conditions, as shown in Eq. 23. 


$$
\begin{aligned}
& O C V_{k}=3.205 * S^{\prime} O C_{\mathrm{k}}{ }^{7}+17.73 * \operatorname{SOC}_{\mathrm{k}}{ }^{6}-76.58 * \operatorname{SOC}_{\mathrm{k}}{ }^{5}+99.04 * \operatorname{SOC}_{\mathrm{k}}{ }^{4}-55.56 * \operatorname{SOC}_{\mathrm{k}}{ }^{3} \\
& +13.54 * S_{\mathrm{k}}{ }^{2}-0.6089 * \mathrm{SOC}_{\mathrm{k}}+3.425
\end{aligned}
$$

Among them, SOCk represents the battery SOC value at the current moment k; OCVk represents the fitted voltage of the battery at the current moment $\mathrm{k}$. The algorithm based on AFFRLS abandons the tedious calculation of traditional offline parameter identification and can realize fast-tracking of dynamic model changes. Effective analysis of vehicle dynamic test conditions can facilitate the rapid correction of the algorithm, thereby improving the accuracy of the algorithm. The online parameter changes of BBDST and DST are shown in Fig. 7.

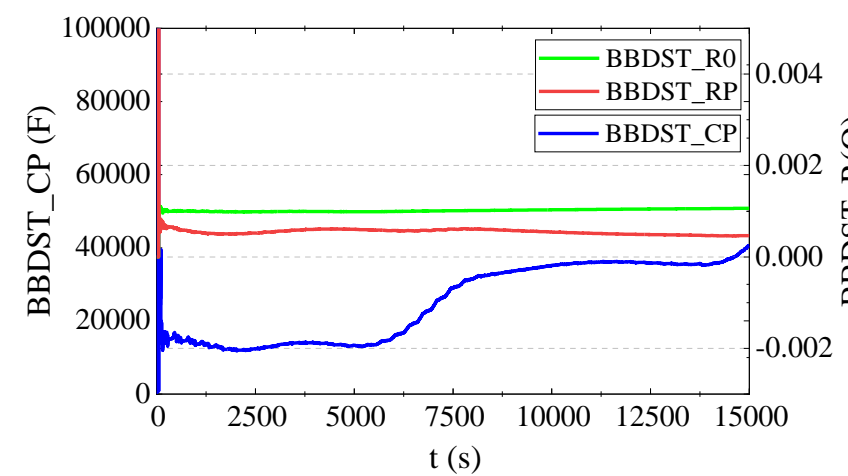

(a) Online parameter identification curve based on BBDST

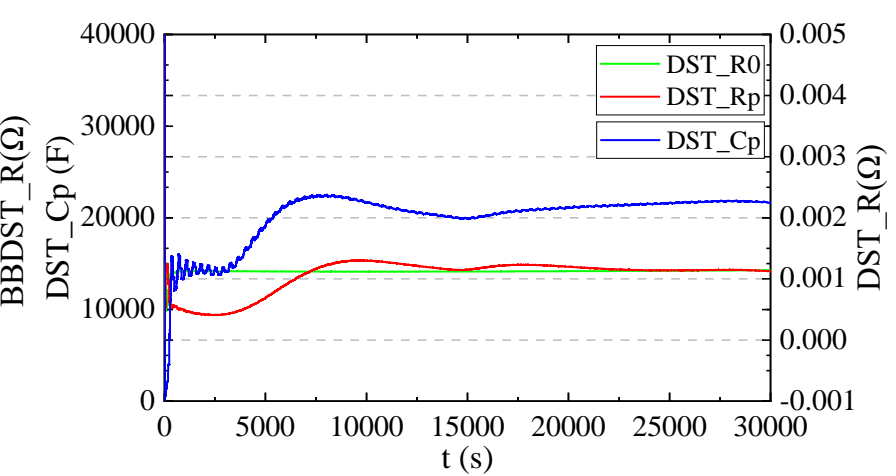

(b) Online parameter identification curve based on DST

Figure 7. Online parameter identification curve analysis

It can be seen from the figure that the ohmic resistance of the battery tends to be stable at about $0.001 \Omega$; the polarization resistance of the battery is more dynamically disturbed and fluctuates; the polarization capacitance of the battery fluctuates greatly, which is in a reasonable range.

All excellent system identification structures are inseparable from the accurate description of the data. According to the work described above, the simulation description of the lithium-ion battery under different working conditions is analyzed. The online simulation error representation of the lithium-ion battery under the time domain working conditions can be respectively represented in Fig. 8 .

It is undeniable that figures (a) and (b) are based on the online simulation demonstration effect of BBDST and DST conditions, respectively. It can be seen from Fig. 8 that the online simulation error remains within $0.03 \mathrm{~V}$ for a long time. Online parameter identification can realize high-precision estimation and provide high-precision input variables for SOP estimation.

The state of the energy consumption effect of the battery in the charging or discharging state is different. SOP estimation under different conditions is essential. It is not advisable to analyze the charging power expression for the battery working condition that is discharged for a long time. Therefore, the following will only study the power representation of the battery's discharge process. 

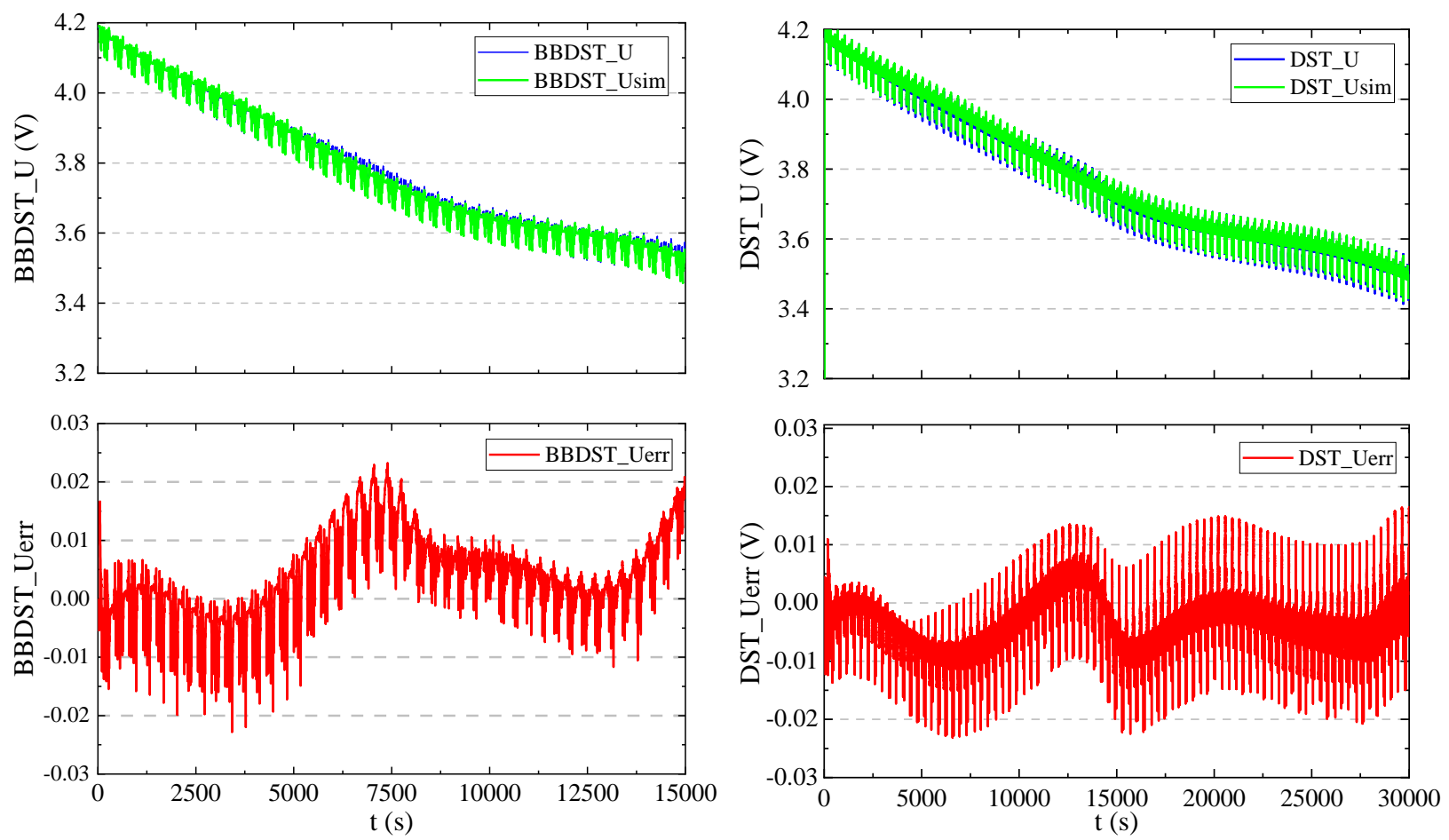

(a) Voltage comparison curve and voltage error curve of BBDST working condition

(b) Voltage comparison curve and voltage error curve of DST working condition

Figure 8. Voltage comparison curve and error curve of online parameter identification based on AFFRLS

According to the BBDST experimental conditions and DST experimental conditions, the SOP characteristics of the lithium-ion battery in the charge and discharge state are analyzed, as shown in Fig. 9 (a) and (c). Combined with the above work, the demonstration effects of different constraint estimation methods are different. Among them, the SOP error under restricted conditions is shown in Fig. 9(b) and (d).

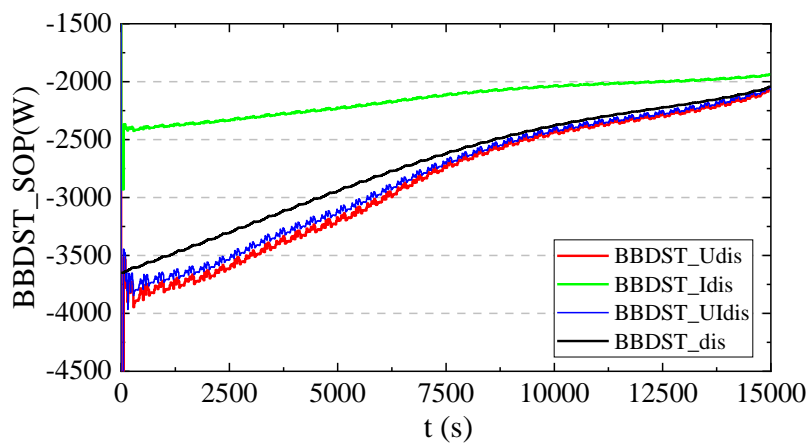

(a) SOP curve with different limits based on BBDST

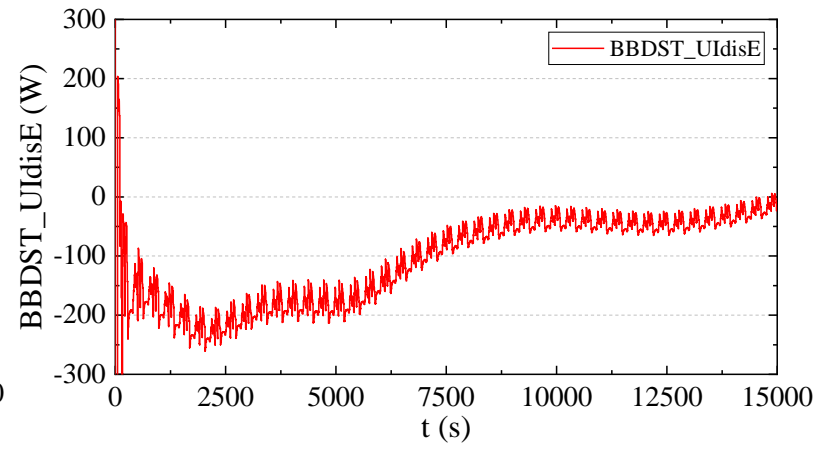

(b) SOP error curve based on different limits of BBDST 


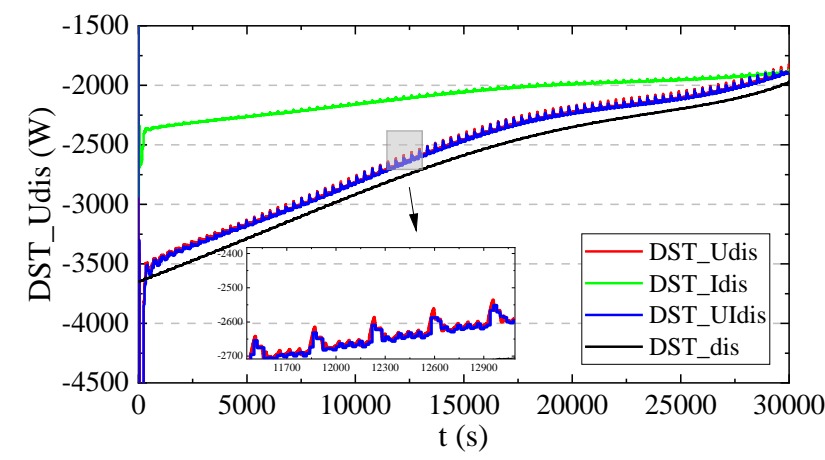

(a) SOP curve with different limits based on DST

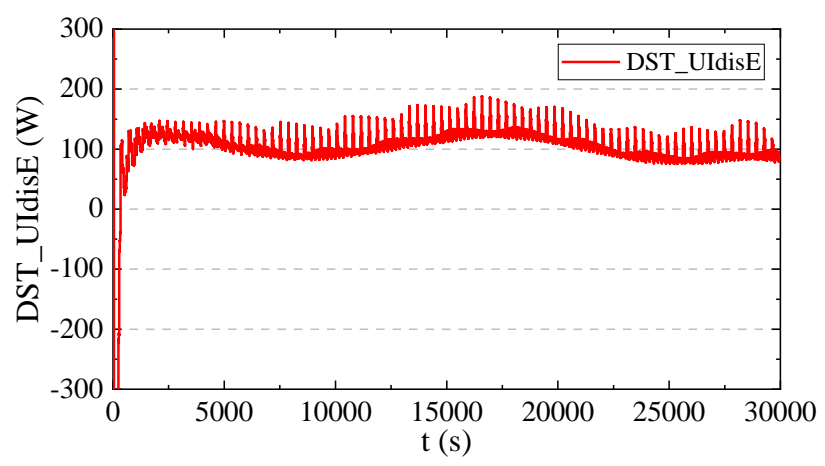

(b) SOP error curve based on different limits of DST

Figure 9. SOP estimation curve and its error curve based on different limits of dynamic stress conditions

Among them, Udis means SOP estimation based on voltage limit; Idis means SOP estimation based on the current limit; UI means SOP estimation considering time scale fusion model limitation; dis means reference quantity obtained by traditional HPPC method (the peak discharge duration of the HPPC test is 10s). Obviously, the estimated value and the actual value of the SOP based on the voltage limit tend to be approximately parallel; the estimated value and the actual value of the SOP based on the current limit tend to cross, and the fusion model based on the voltage and current limit can integrate the advantages of the two methods and effectively fit the actual changes. The SOP relative error curves of the fusion models based on different working conditions are shown in Fig. 10.

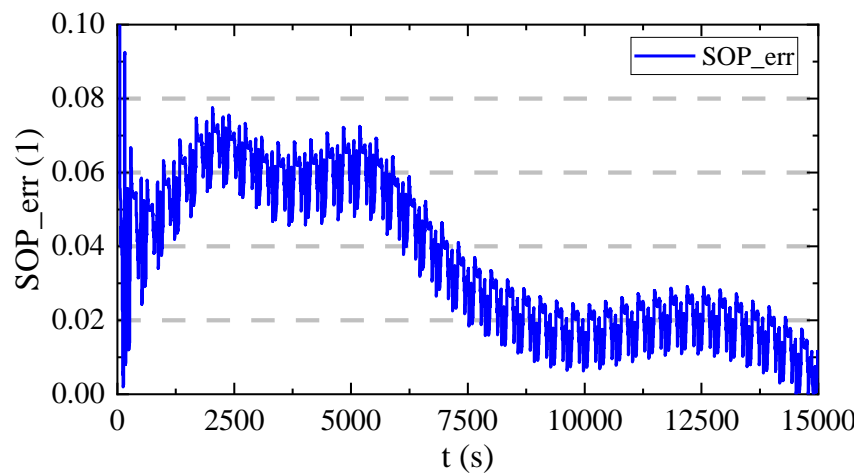

(a) SOP relative error curve of fusion model based on BBDST working condition

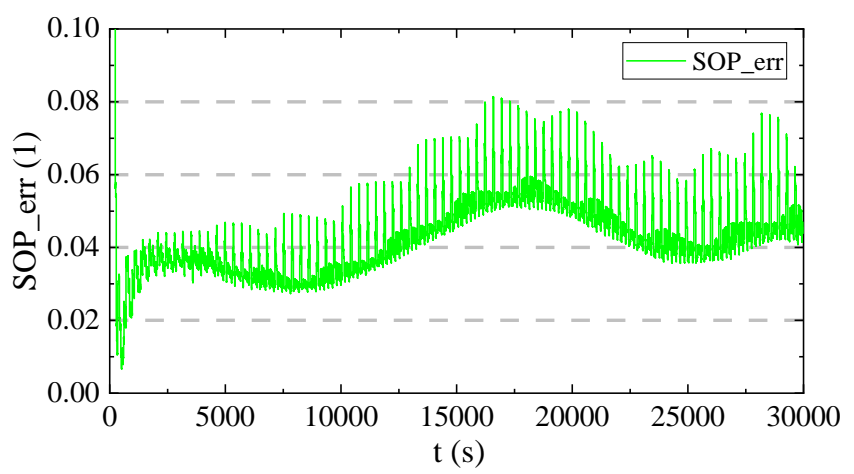

(b) SOP relative error curve of fusion model based on DST working condition

Figure 10. SOP relative error curves of fusion models based on different working conditions

Where the data shown in Fig. 10 represent the relative error between the error of the SOP of the battery under the fusion model and the theoretical difference of the 10sHPPC pulse discharge. As can be seen from the figure, the error of the algorithm is less than $8 \%$ for both operating conditions, which enables a reliable prediction of the power. 


\section{DISCUSSIONS}

The basis for the above algorithm to enable SOP prediction is the ability to build an adaptive parameter identification system based on dynamic parameter changes. Therefore, it is feasible to analyze the power state of the battery by considering the impact of different parameter identification methods on the battery description, using the voltage and SOC of the established battery to simulate the limits of the battery. The description of the condition by presetting the sampling time is not conducive to analyze its power variation at each sampling time length for a condition that varies singularly throughout the cycle.

The analysis of the constituent components for different batteries whose discharging conditions are useful for quantitative description for complex conditions. This study is an extension of it and facilitates the description of the battery power output characteristics at long time. The method has higher adaptability and robustness, and higher accuracy for long time estimation [32]. It also has a lower computational complexity and a smaller loss of accuracy of about $2 \%$ [33].

\section{CONCLUSIONS}

Lithium-ion battery state of power is a reliable factor to characterize the battery state. In this study, the analysis of charge and discharge constitutive factors under-sampling time is established, and the online identification of parameters is realized by establishing an adaptive forgetting factor recursive least-squares method based on the Thevenin model, and the effective description of the SOP under voltage and current limitations is achieved by using the online parameters. Firstly, by analyzing the battery model to construct the adaptive online parameter identification, a reliable estimation of the battery is achieved with a voltage error lower than $0.03 \mathrm{~V}$. Secondly, by analyzing the charge and discharge components of the battery under the sampling time and setting the coupling reference quantity to judge the power occupancy, an effective analysis of the SOP is completed. Finally, a fusion model based on voltage and current limits is constructed to achieve an effective tracking of the SOP, in which the power error of the battery is lower than $8 \%$.

\section{ACKNOWLEDGMENTS}

The work was supported by the National Natural Science Foundation of China (No. 61801407).

\section{References}

1. M. Zhao, T. Sun and Q. Feng, Science of the Total Environment, 784 (2021)147104.

2. M. T. Muhssin, Z. A. Obaid, K. Al-Anbarri, L. M. Cipcigan and M. N. Ajaweed, Int J Elec Power, 130 (2021)106920.

3. H. Dong, Q. Gong and M. Zhu, Int J Elec Power, 130 (2021)106910.

4. Y. Zhang, Y. Huang, H. Chen, X. Na, Z. Chen and Y. Liu, Energy, 228 (2021)120568.

5. H. Xu, R. Feng, Z. Wang, N. Zhang, R. Zhang, K. He, Q. Wang, Q. Zhang, J. Sun, B. Zhang, Z. Shen, S. H. S. Ho and J. Cao, Environmental Pollution, 282 (2021)117057.

6. $\quad$ B. Lai, P. Yi, Y. Sui and Q. Zhang, Neurocomputing, 444 (2021) 179. 
7. S. Ding, R. Li and S. Wu, Communications in Nonlinear Science and Numerical Simulation, 99 (2021) 105847.

8. $\quad$ X. Liang, P. Zhang, Y. Fang, H. Lin and W. He, Ieee T Ind Electron, 68 (2021) 6020.

9. $\quad$ A. Ibrahim and F. Jiang, Renew Sust Energ Rev, 144 (2021)111049.

10. W. Chen, C. Xu, M. Chen, K. Jiang and K. Wang, J Energy Storage, 36 (2021)102387.

11. P. Lin, P. Jin, J. Hong and Z. Wang, Energy Rep, 6 (2020) 2299.

12. K. L. Muh, A. C. Caliwag, I.-S. Jeon and W. Lim, The Journal of Korean Institute of Communications and Information Sciences, 46 (2021) 314.

13. T. Zhang, N. Guo, X. Sun, J. Fan, N. Yang, J. Song and Y. Zou, Sustainability, 13 (2021)5166.

14. Y. Z. Gao, X. Zhang, B. J. Guo, C. Zhu, J. Wiedemann, L. Wang and J. H. Cao, Ieee T Ind Inform, 16 (2020) 3417.

15. W. Zhou, R. J. Huang, K. Liu and W. G. Zhang, Int J Energ Res, 44 (2020) 3558.

16. F. Roder, V. Laue and U. Krewer, Batteries Supercaps, 2 (2019) 248.

17. Y. Q. Tan, M. J. Luo, L. Y. She and X. Y. Cui, Int J Electrochem Sc, 15 (2020) 1128.

18. X. Lai, S. Y. Wang, S. D. Ma, J. Y. Xie and Y. J. Zheng, Electrochim Acta, 330 (2020)135239.

19. U. Morali and S. Erol, Turk J Chem, 44 (2020) 602.

20. X. Yang, L. Chen, X. Xu, W. Wang, Q. L. Xu, Y. Z. Lin and Z. G. Zhou, Energies, 10 (2017)1811.

21. X. Zhang, J. L. Lu, S. F. Yuan, J. Yang and X. Zhou, J Power Sources, 345 (2017) 21.

22. N. Chen, P. Zhang, J. Y. Dai and W. H. Gui, Ieee Access, 8 (2020) 26872.

23. H. Khalifa, P. S. A. El-Safty, A. Reda, M. A. Shenashen, A. Elmarakbi and H. A. Metawa, Batteries Supercaps, 3 (2020) 76.

24. S. L. Wang, C. Fernandez, Y. C. Fan, J. Q. Feng, C. M. Yu, K. F. Huang and W. Xie, Energy Sci Eng, 8 (2020) 1484.

25. F. Z. Wen, B. Duan, C. H. Zhang, R. Zhu, Y. L. Shang and J. M. Zhang, Electronics-Switz, 8 (2019)838.

26. H. Pang, L. J. Mou and L. Guo, Chinese Phys B, 28 (2019)108201.

27. S. L. Wang, C. Fernandez, L. P. Shang, Z. F. Li and H. F. Yuan, T I Meas Control, 40 (2018) 1892.

28. X. Lai, L. He, S. Y. Wang, L. Zhou, Y. F. Zhang, T. Sun and Y. J. Zheng, J Clean Prod, 255 (2020)120203.

29. Z. Cheng, Y. H. Xing, S. L. Cheng and P. Y. Guo, Cluster Comput., 22 (2019) S8553.

30. $\quad$ L. Yang, Y. S. Cai, Y. X. Yang and Z. W. Deng, Appl Energ, 257 (2020)114006.

31. L. Bowen, W. Shunli, F. Carlos, Y. Chunmei and F. Yongcun, J Energy Storage, 40(2021)102583.

32. C. Wei, M. Benosman and T. Kim, Int J Control Autom, 17 (2019) 2906.

33. P. Lin, P. Jin, J. C. Hong and Z. P. Wang, Energy Rep, 6 (2020) 2299.

(C) 2021 The Authors. Published by ESG (www.electrochemsci.org). This article is an open access article distributed under the terms and conditions of the Creative Commons Attribution license (http://creativecommons.org/licenses/by/4.0/). 